\title{
Facile Strategy for the Biomimetic Heterogeneous Design of Elastomers with Mechanical Robustness, Malleability, and Functionality
}

\author{
Jing Huang, ${ }^{\dagger}$ Shaoxin Kong, ${ }^{\dagger}$ Zhenghai Tang, ${ }^{\dagger}$ Siwu Wu, ${ }^{+}$Baochun Guo, ${ }^{*,+}$ and Liqun Zhang ${ }^{*,+}$ \\ + Department of Polymer Materials and Engineering, South China University of Technology, Guangzhou 510640, China \\ ₹ State Key Laboratory of Organic/Inorganic Composites, Beijing University of Chemical Technology, Beijing 100029, China \\ *E-mail: psbcguo@scut.edu.cn \\ *E-mail: zhanglq@mail.buct.edu.cn
}

\section{Experimental Section}

Materials. ENR, 50\% epoxidization degree, was supplied by the Agricultural Products Processing Research Institute, Chinese Academy of Tropical Agricultural Science, China. Sebacic acid (SA, 99\%), 1,2dimethylimidazole (DMI, 99\%), and zinc acetate $\left(\mathrm{Zn}(\mathrm{Ac})_{2} \cdot 2 \mathrm{H}_{2} \mathrm{O}, 98 \%\right)$ were purchased from Sigma-Aldrich. Multi-walled carbon nanotubes 9100 (MWCNTs, purity $\geq 97.5 \%$ ) were provided by CNano Technology Limited, Beijing, China. Precipitated silica sil-380B (specific surface area of $180 \mathrm{~m}^{2} / \mathrm{g}$ ) was kindly supplied by NanPing Jialian Chemicals, China.

Preparation of the hetero-VEs. Firstly, the hard vitrimer powders with relatively high cross-linking density were prepared. Specifically, $9.5 \mathrm{wt} \%$ (relative to ENR, hereinafter) SA, $2.05 \mathrm{wt} \%$ (10 mol \% relative to the carboxyl group) $\mathrm{Zn}(\mathrm{Ac})_{2} \cdot 2 \mathrm{H}_{2} \mathrm{O}$ and $1 \mathrm{wt} \%$ DMI were successively compounded with ENR gum by using a two-roll mill for about $15 \mathrm{~min}$. The stoichiometry of the oxirane and carboxyl groups was 7 . After mixing, the compounds were subjected to compression at $180{ }^{\circ} \mathrm{C}$ for the optimum curing time determined by a vulcameter. Next, the prepared hard ENR vitrimer were grinded on a two-roll mill. After grinding, the powder were compounded with ENR gum, $1.9 \mathrm{wt} \% \mathrm{SA}, 0.41 \mathrm{wt} \% \mathrm{Zn}(\mathrm{Ac})_{2} \cdot 2 \mathrm{H}_{2} \mathrm{O}$ and $1 \mathrm{wt} \% \mathrm{DMI}$ by using the same procedures as above used. The weight ratio of ENR gum in the soft matrix and ENR gum in the hard phases is variable, hence, the total amount of SA in hetero-VE is varied with the weight ratio of ENR gum in soft 
matrix and ENR gum in hard phases varied. In the context, the samples are coded as hetero- $x$, which refers to the total amount of SA is $x$ wt $\%$ relative to the total amount of ENR.

\section{Preparation of the homo-VEs.}

The control samples that are homogeneously cross-linked, coded as homo- $x$, where $x$ refers to the amount of SA, are prepared by successively compounding desired amounts of $\mathrm{SA}, \mathrm{Zn}(\mathrm{Ac})_{2} \cdot 2 \mathrm{H}_{2} \mathrm{O}$ and DMI with ENR gum by using a two-roll mill for about $15 \mathrm{~min}$. After mixing, the compounds were subjected to compression at $180^{\circ} \mathrm{C}$ for the optimum curing time determined by a vulcameter.

Preparation of the hetero-H-VEs and hetero-S-VEs. The preparation procedures are the same as hetero- $x$. For hetero-H-VEs, the weight ratio of ENR gum in soft matrix and ENR gum in hard ENR is fixed to be 60/40, and the SA content is varied in the hard phase. These samples are labeled as hetero-H- $y$, referring to $y$ wt\% SA in the whole sample. For hetero-S-VEs, the weight ratio of ENR gum in soft matrix and ENR gum in hard ENR is fixed to be $20 / 80$, and the SA content is varied in the soft matrix. These samples are labeled as hetero-S-z, referring to $z$ wt\% SA in the whole sample.

Preparation of the hetero-9.5-CNT-VEs. To have a distinct volume exclusion effects, the weight ratio of ENR gum in soft matrix and ENR gum in hard ENR is fixed to be 20/80. Firstly, ENR/MWCNTs master batch containing $50 \mathrm{wt} \%$ MWCNTs was prepared according to our previously publication, ${ }^{1}$ in order to improve the dispersion of MWCNTs. After then, the ENR/MWCNTs master batch were successively compounded with ENR gum, hard ENR powder, $\mathrm{SA}, \mathrm{Zn}(\mathrm{Ac})_{2} \cdot 2 \mathrm{H}_{2} \mathrm{O}$ and DMI and compressed similar as hetero-9.5. The samples are coded as hetero-9.5-zCNT, referring to $z$ wt\% MWCNTs in the sample. Likewise, the control samples of homogeneously dispersed SA and MWCNTs were prepared and coded as homo-9.5$z \mathrm{CNT}$

The main formulas of all the samples are listed in Table S1. 
Welding experiment. Two parts of hard ENR vitrimer and a part of soft ENR compound (with gum, curing agents and catalysts) (Figure S1) were pressed at $180{ }^{\circ} \mathrm{C}$ for $10 \mathrm{~min}$ and $20 \mathrm{~min}$, respectively, which is analogous to the curing process of the hetero-VE samples. The formulas of the hard phase and soft phase are based on the sample hetero-5.7 (Table S1). After that, the samples underwent tensile tests and their fracture behaviors were recorded by video. Three specimens for tensile tests for each sample.

\section{Characterizations.}

A tecnai G2 F30 S-Twin electron microscope was used to collect transmission electron microscopy (TEM) images for the ultramicrotomed samples. The cryogenically fractured surface of the samples was observed using scanning electron microscopy (SEM, Hitachi S-4800). DMT Modulus maps were measured under ambient conditions in the PeakForce quantitative nano-mechanical mapping (QNM) mode by a Multimode 8 AFM retrofitted with the NanoScope V controller (Bruker). PeakForce Tapping was done with silicon tips coated by $\mathrm{Ti} / \mathrm{Au}$ on a nitride lever (SNL-10, Bruker Nano Inc) with a spring constant of $0.24 \mathrm{~N} / \mathrm{m}$ and tip radius of $5 \mathrm{~nm}$. PeakForce Tapping oscillates the sample at $60 \mathrm{kHz}$. The working distance was set to $150 \mathrm{~nm}$. U-CAN UR-2030 vulcameter was used to determine the curing characteristics at $180^{\circ} \mathrm{C}$.

Fourier transform infrared spectroscopy (FTIR) was collected on a Bruker Vertex 70 FTIR spectrometer equipped with a heating cell.

Cross-linking density was determined by equilibrium swelling experiment in toluene based on Flory-Rehner equation. ${ }^{2}$ Preweighed vulcanizates were swollen in toluene at room temperature for $72 \mathrm{~h}$, and the solvent is replaced with fresh solvent for each $24 \mathrm{~h}$. After swelling, the solvent was wiped off quickly from the sample surface using filter paper, and the samples were immediately weighed and then dried in a vacuum oven at $60^{\circ} \mathrm{C}$ until constant weight. Three specimens were measured for each sample.

The volume fraction of rubber in the swollen gel $\left(V_{r}\right)$ was calculated according to the following equation:

$$
V_{r}=\frac{\left(m_{2}-m_{0} \varphi\right) / \rho_{r}}{\left(m_{2}-m_{0} \varphi\right) / \rho_{r}+\left(m_{1}-m_{0}\right) / \rho_{s}}
$$


where $m_{0}$ is the weight of the sample before swelling; $m_{1}$ and $m_{2}$ are the weights of the swollen and dried sample, respectively; $\varphi$ is the weight fraction of the insoluble components. $\rho_{r}$ and $\rho_{s}$ are the densities of the rubber and solvent, respectively.

The elastically active network chain density can be calculated by the well-known Flory-Rehner equation:

$V_{e}=-\frac{\ln \left(1-V_{r}\right)+V r+\chi V_{r}^{2}}{V_{S}\left(V_{r}^{1 / 3}-V_{r} / 2\right)}$

where $V_{r}$ is the volume fraction of polymer in the swollen sample, $\chi$ is the Flory-Huggins polymer solvent interaction parameter ( 0.341 for ENR and toluene), and $V_{S}$ is the molar volume of the solvent $\left(107 \mathrm{~cm}^{3} / \mathrm{mol}\right.$ for toluene).

Tensile tests and loading-unloading tests performed on dogbone-shaped samples (ca. $75 \mathrm{~mm} \times 4 \mathrm{~mm} \times 0.5$ $\mathrm{mm}$ and a gauge length of $20 \mathrm{~mm}$ ) with an extension rate of $500 \mathrm{~mm} / \mathrm{min}$ at room temperature. Five specimens for tensile tests were measured for each sample, and the average value and standard deviation were calculated. For comparison of loading-unloading cycle without and with prestretching, the samples were first stretched to a strain of $150 \%$ (100\% for hetero-9.5 to avoid break) and then underwent unloading. Next, the samples were kept at $150 \%$ or $100 \%$ for $20 \mathrm{~min}$ and then were allowed to relax at ambient temperature for 20 min. After that, the samples underwent another loading-unloading cycle. Loading-unloading curves at $90{ }^{\circ} \mathrm{C}$ were collected by using a TA DMA Q 800 with a strain rate of $500 \% / \mathrm{min}$. As the samples were too rigid at 25 ${ }^{\circ} \mathrm{C}$ to conduct tests on the TA DMA Q800, the corresponding loading-unloading tests at $25^{\circ} \mathrm{C}$ were carried out on the U-CAN UT-2060 with a strain rate of 500\%/min.

Dynamic mechanical analysis (DMA), stress relaxation tests and shape memory measurements were performed on rectangular specimen $(10 \mathrm{~mm} \times 4 \mathrm{~mm} \times 0.5 \mathrm{~mm})$ by using a TA-Q800 DMA apparatus. For DMA investigations, a tensile mode was selected with the frequency of $1 \mathrm{~Hz}$ and the strain of $0.5 \%$. The sample was scanned from -50 to $150{ }^{\circ} \mathrm{C}$ at a heating rate of $3{ }^{\circ} \mathrm{C} / \mathrm{min}$. For stress relaxation tests, the samples were 
relaxed at a constant strain of $5 \%$. A thermal equilibrium procedure was conducted at the required temperature for $15 \mathrm{~min}$ before the stress decay was recorded. To investigate the shape memory behavior, the samples were firstly stretched to a set stress of $0.5 \mathrm{MPa}$ with a stress ramping rate of $0.5 \mathrm{MPa} / \mathrm{min}$ after a thermal equilibrium at $90^{\circ} \mathrm{C}$. Subsequently, the samples were cooled to $0{ }^{\circ} \mathrm{C}$ at a rate of $10^{\circ} \mathrm{C} / \mathrm{min}$ to fix the deformation, yielding a setting strain of $\varepsilon_{s}$. After that, the stress was unloaded at $0.5 \mathrm{MPa} / \mathrm{min}$, followed by an additional $15 \mathrm{~min}$ of isothermal step to ensure shape fixing, leaving a fixing strain $\varepsilon_{\text {f. }}$ The permanent shape was recovered by heating at $10{ }^{\circ} \mathrm{C} / \mathrm{min}$ to $90{ }^{\circ} \mathrm{C}$ for $15 \mathrm{~min}$ and the final stain was $\varepsilon_{\mathrm{r}}$. The permanent shape was reprogrammed by stretching the samples with a constant stress of $0.5 \mathrm{MPa}$ for $45 \mathrm{~min}$ at $180^{\circ} \mathrm{C}$ and then the stress was released to recover the elastic deformation for $10 \mathrm{~min}$. Subsequently, a new shape memory cycle was executed same as described above.

The shape fixity $\left(R_{f}\right)$ and recovery $\left(R_{r}\right)$ are calculated as follows:

$$
\begin{aligned}
& \mathrm{R}_{\mathrm{f}}=\frac{\varepsilon_{\mathrm{f}}-\varepsilon_{\mathrm{r}}}{\varepsilon_{\mathrm{s}}-\varepsilon_{\mathrm{r}}} \times 100 \% \\
& \mathrm{R}_{\mathrm{r}}=\frac{\varepsilon_{\mathrm{s}}-\varepsilon_{\mathrm{r}}}{\varepsilon_{\mathrm{s}}-\varepsilon_{\mathrm{r}}} \times 100 \%
\end{aligned}
$$

Two types of characterization apparatus were used in evaluating the electrical conductivity of the prepared samples depending on the conductivity of the samples. For the homo-9.5-zCNT with high resistivity, volume resistivities were measured at room temperature using Keithley $6517 \mathrm{~B}$ electrometer/high resistance meter with an 8009 resistivity test fixture. For the hetero-9.5-zCNT samples with high conductivity, electrical properties were measured at room temperature using Keithley 2635A sourcemeter with a two-probe method.

\section{Figures and Tables}




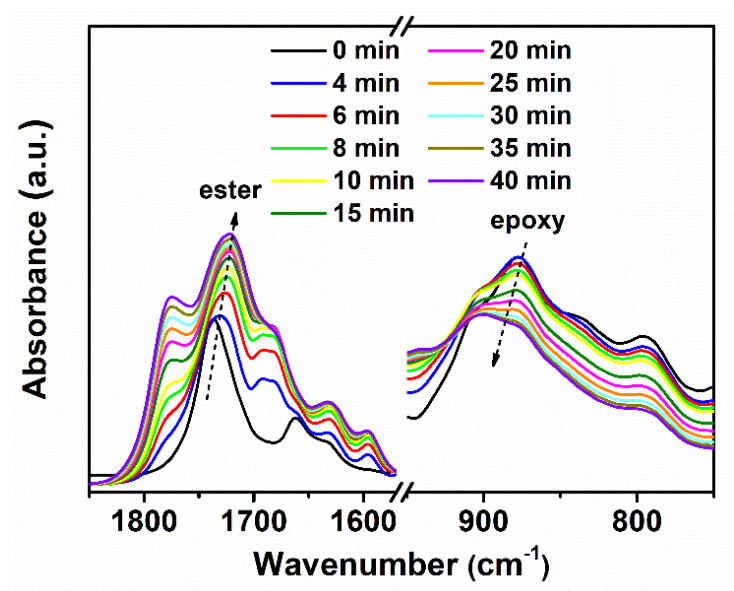

Figure S1. Evolutions of carboxyl groups $\left(1850-1570 \mathrm{~cm}^{-1}\right)$ and epoxy group $\left(950-750 \mathrm{~cm}^{-1}\right)$ of FTIR spectra during the curing of homo- 3.8 at $180{ }^{\circ} \mathrm{C}$.

As the curing proceeds, the absorption for epoxy group at $877 \mathrm{~cm}^{-1}$ gradually decreases while that for ester groups at $1723 \mathrm{~cm}^{-1}$ gradually increases. The traces showed that the reaction was complete within $30 \mathrm{~min}$.
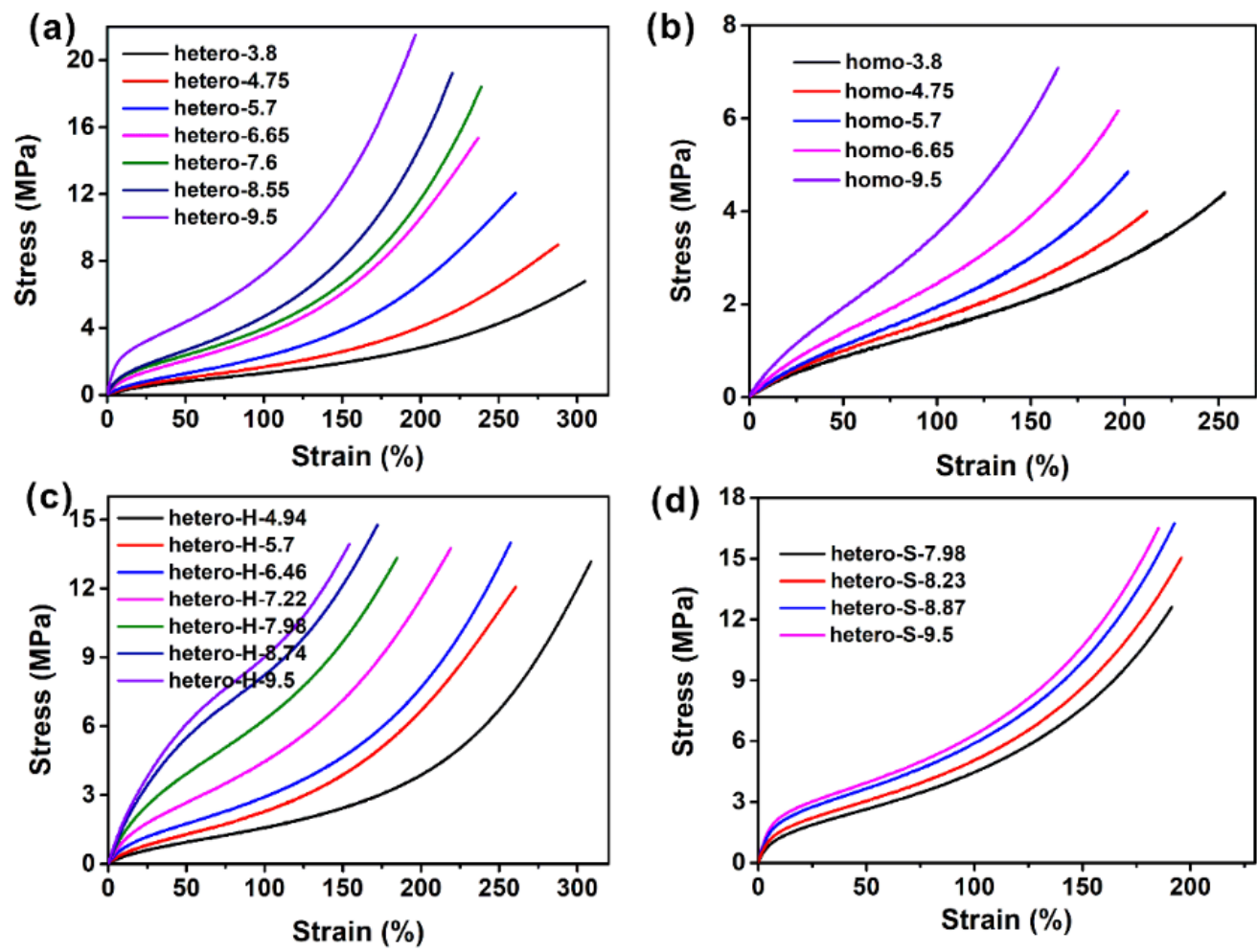

Figure S2. Stress-strain curves of (a) hetero-VEs (SA content in hard phases is fixed to be $9.5 \mathrm{wt} \%$ and ENR ratio of soft and hard phase varies) (b) homo-VEs (SA is homogeneously dispersed and SA content varies) (c) hetero-H-VEs (ENR ratio of soft and hard phase is fixed to be 60/40 and SA content in hard phases varies) and (d) hetero-S-VEs (ENR ratio of soft and hard phase is fixed to be $20 / 80$ and SA content in soft matrix varies). 


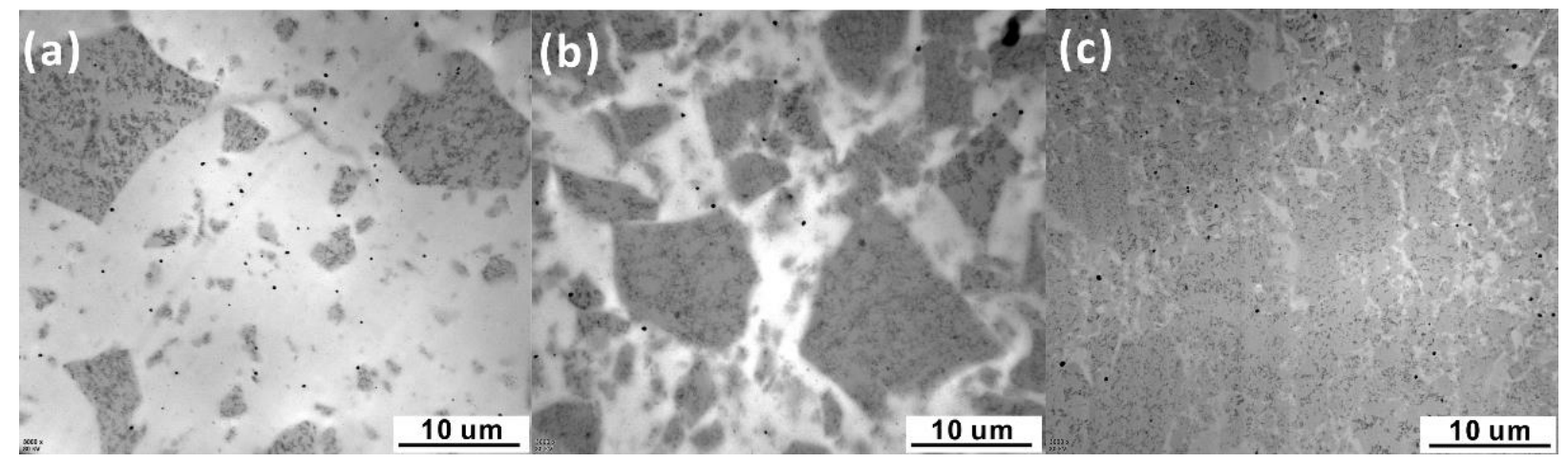

Figure S3. TEM images of (a) hetero-3.8, (b) hetero-6.65, and (c) hetero-9.5 with $5 \mathrm{wt} \%$ silica incorporated into the hard phase to distinguish the soft matrix and hard phases more clearly.
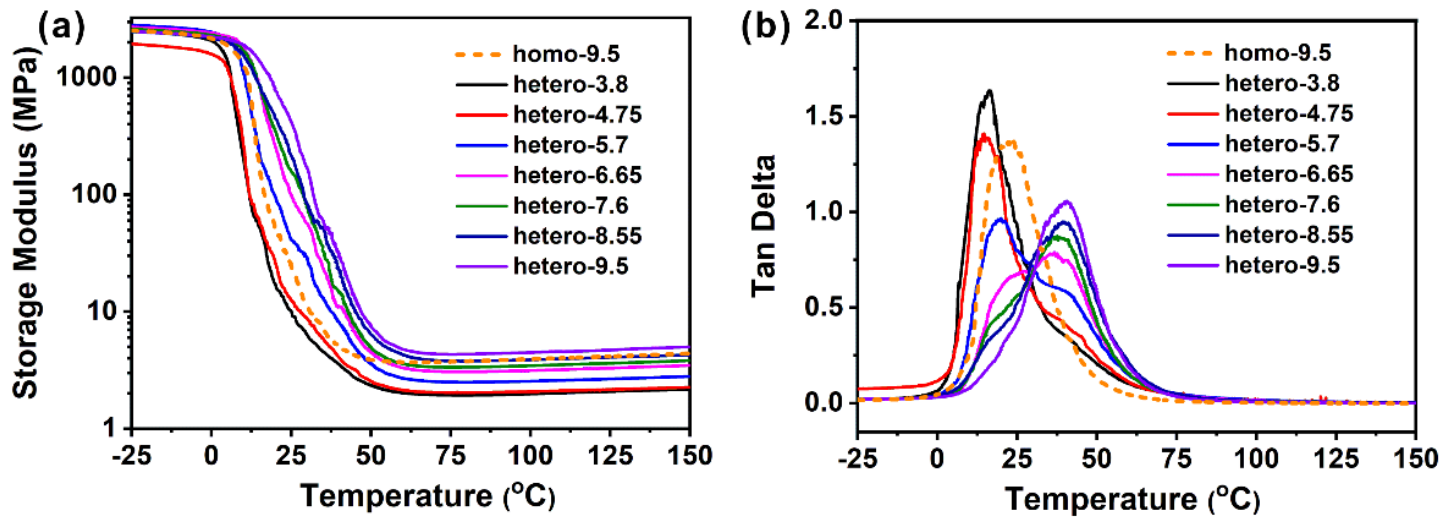

Figure S4. Dependence of (a) storage modulus and (b) loss factor ( $\tan \delta$ ) on temperature for homo-9.5 and hetero-VEs.

Two distinct loss peaks can be found in the hetero-VEs, which are related to the glass transition of the soft matrix and hard phases. When increasing the hard phase content, the storage modulus increases continuously, the loss peak height at low temperatures depresses, and that at high temperatures becomes more intense. In addition, the glass transition temperature $(\mathrm{Tg})$ of the soft matrix gradually shifts to elevated temperatures due to increased constraints from an increased number of cross-links and hard phases.
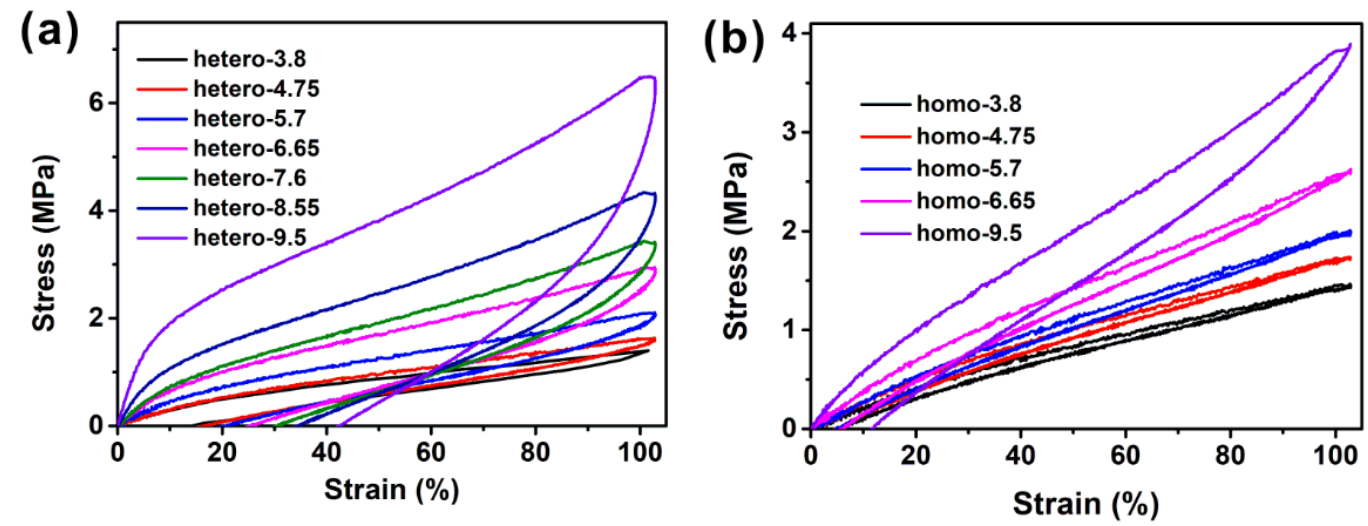
Figure S5. Loading-unloading curves of (a) hetero-VEs and (b) homo-VEs.

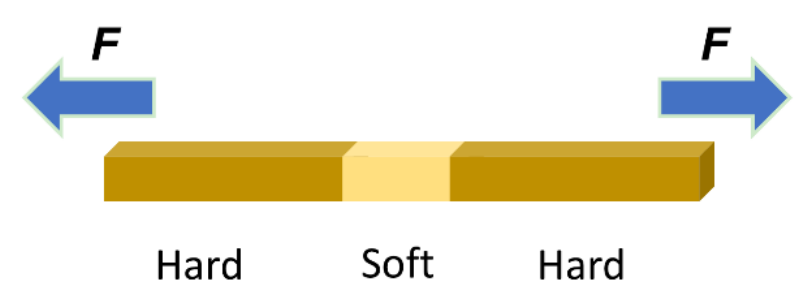

Figure S6. Schematic illustration of the welded samples.

From the movie $\mathbf{S 1}$ and $\mathbf{S 2}$, it can be seen that for the sample that welded for $10 \mathrm{~min}$, as the interfacial transesterification and cocross-linking reactions didn't proceed long enough, the interfacial interactions are relatively weak, the sample fractured at the interface. When the sample was welded for $20 \mathrm{~min}$ (the optimum curing time of the soft phase is determined as $16 \mathrm{~min}$ by vulcameter), a cohesive fracture, i.e. fracture of soft phase, was found, vividly demonstrating the excellent interfacial interactions between the two phases.

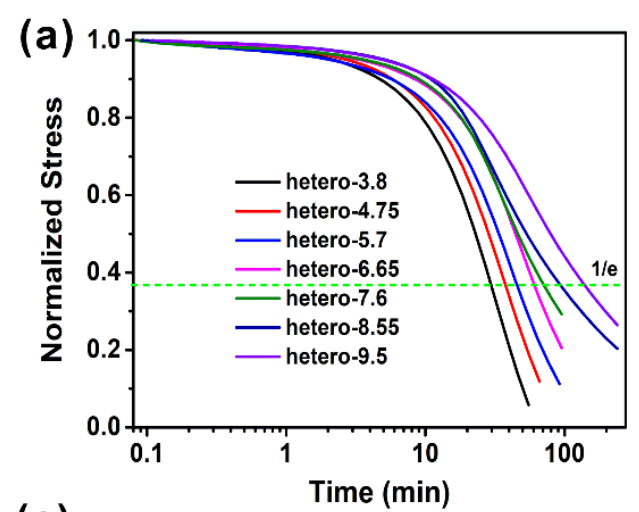

(b)
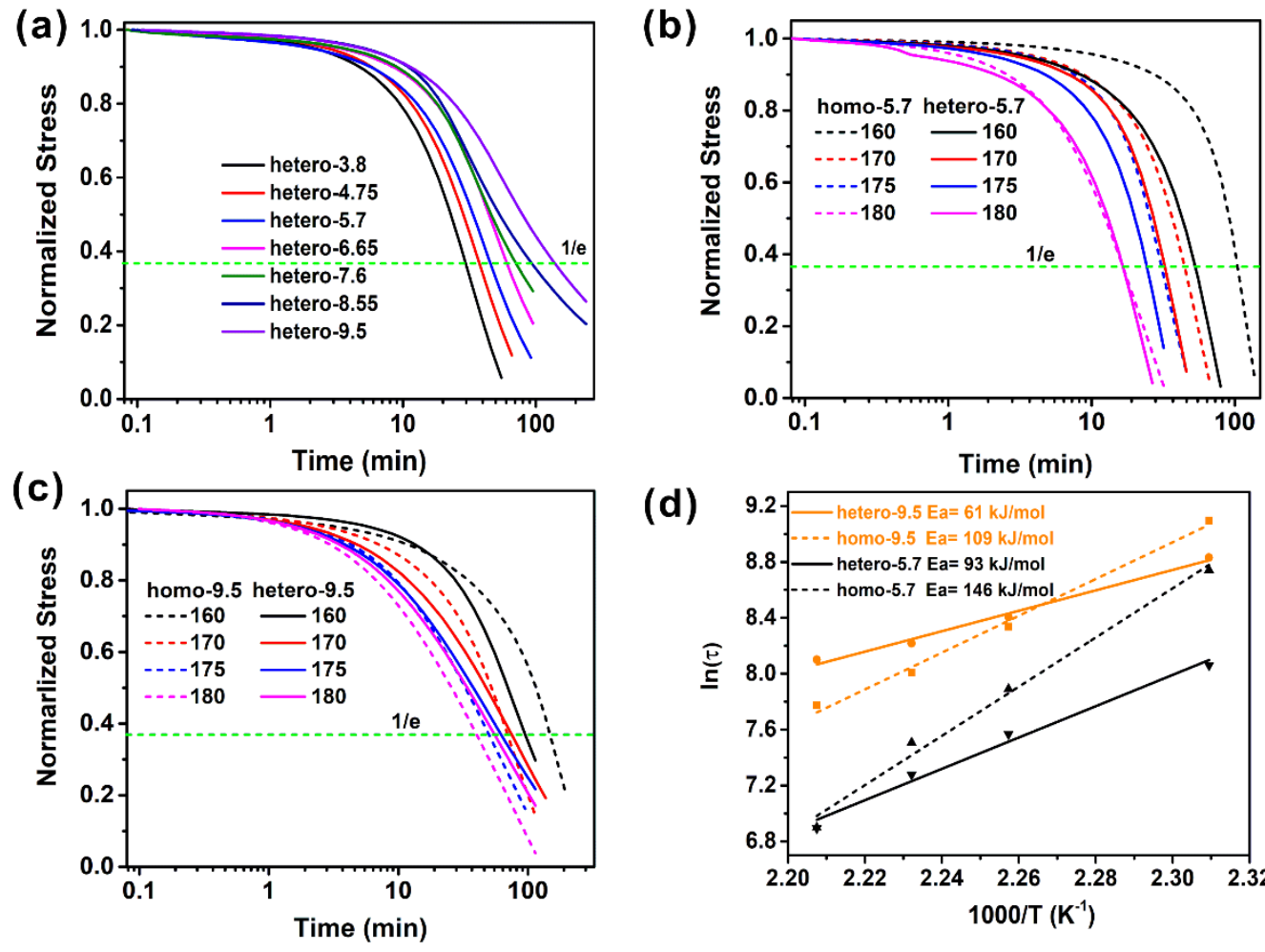

(d)

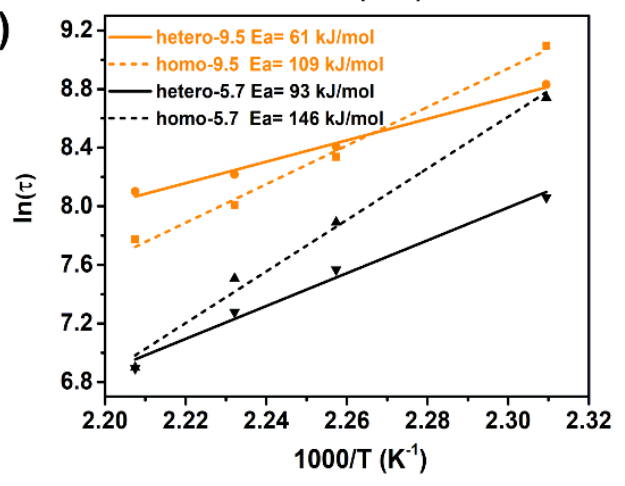

Figure S7. (a) Normalized stress relaxation for hetero-VEs at $180^{\circ} \mathrm{C}$ with a constant strain of $5 \%$. (b) (c) Normalized stress relaxation for hetero-VEs and homo-VEs with SA content of 5.7 and $9.5 \mathrm{wt} \%$ at varied temperatures from $160-180{ }^{\circ} \mathrm{C}$ with a 
constant strain of 2\%. (d) Fitting of $\tau^{*}$ to the Arrhenius equation for hetero-VEs and homo-VEs with SA content of 5.7 and 9.5 wt $\%$.

According to Figure S7d, the activity energy $\left(E_{a}\right)$ for hetero-9.5, homo-9.5, hetero-5.7 and homo-5.7 was calculated to be $61 \mathrm{~kJ} / \mathrm{mol}, 109 \mathrm{~kJ} / \mathrm{mol}, 93 \mathrm{~kJ} / \mathrm{mol}$ and $146 \mathrm{~kJ} / \mathrm{mol}$, respectively. It is interesting to find $\mathrm{E}_{\mathrm{a}}$ of homo-9.5 is much lower than that of homo-5.7 while the relaxation time shows an inverse pattern. This can be attributed to the two-side effects on $\mathrm{E}_{\mathrm{a}}$ and relaxation rate of the cross-linking density. ${ }^{3-5}$ On the one hand, increasing cross-linking density leads to lower chain mobility of rubber, which results in higher energy barrier and slower relaxation rate. On the other hand, a higher concentration of ester group and hydroxyl group with a larger number of cross-links makes the exchange reaction and network rearrangement easier to occur. In the present system, compared with homo-5.7, homo-9.5 has a lower $\mathrm{E}_{\mathrm{a}}$, which indicates the effect of concentration dominates the $\mathrm{E}_{\mathrm{a}}$ in the present cross-linking density range. However, when the temperature is high enough, homo-5.7 has sufficient chain mobility and be able to overcome the confinement of concentration, hence, it relaxes stress faster than homo-9.5 with a poorer chain mobility. Besides, as the cross-linking density of the hard phases in hetero-VEs is higher than that of the corresponding homo-VE counterparts, and the $\mathrm{E}_{\mathrm{a}}$ of a sample should be determined by the part with lower $E_{a}$, the $E_{a}$ of hetero-VEs is lower than that of homo-VEs. Similarly, the relaxation rate of hetero-VEs at high temperature is slower than that of homo-VEs. Another factor that might contribute to the lower $\mathrm{E}_{\mathrm{a}}$ of hetero-VEs is that the hard phases act as a huge cross-linking center with high functionality, which allows ready exchanging reaction between the adjacent ester group and hydroxyl groups. ${ }^{6,7}$ 

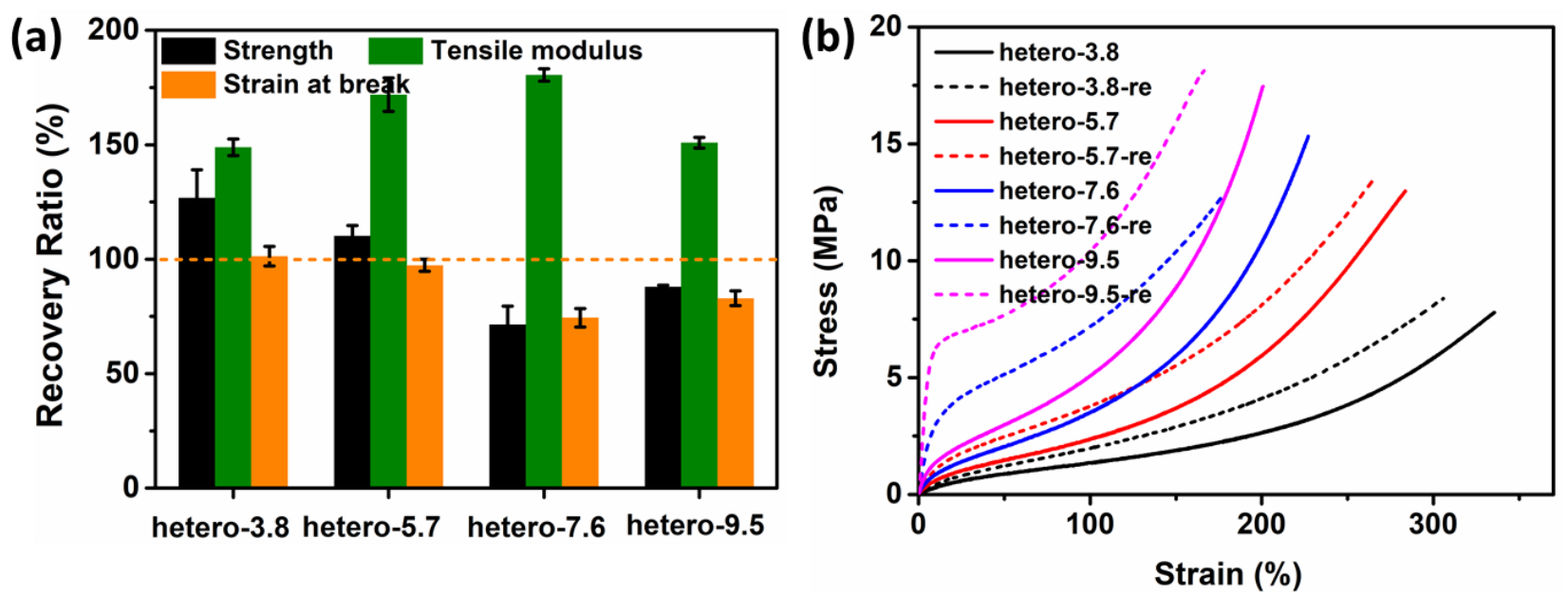

Figure S8. (a) Recovery ratio of tensile strength, tensile modulus, and strain at break of hetero-VEs. (b) Stress-strain curves of hetero-VEs before and after reprocessing.

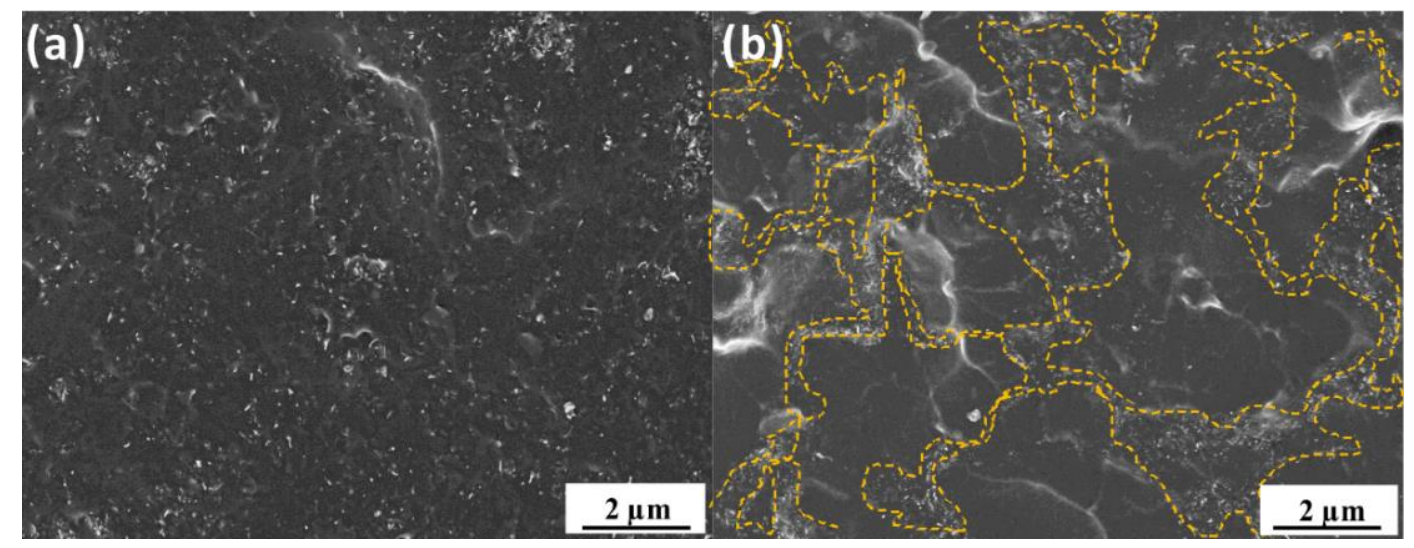

Figure S9. SEM images of homo-9.5-4CNT and hetero-9.5-4CNT.
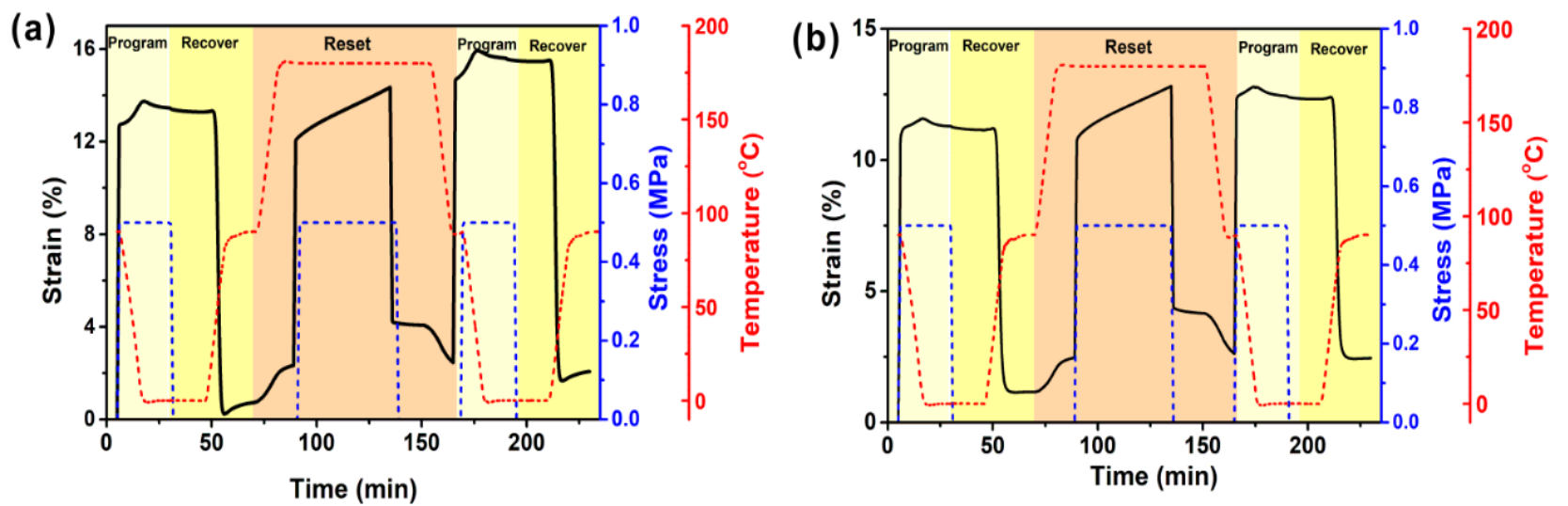

Figure S10. Quantitative shape memory and shape reconfiguration cycles for (a) hetero-9.5 and (b) hetero-9.5-4CNT. 
Table S1. The main formulas of all the hetero samples.

\begin{tabular}{|c|c|c|c|c|c|}
\hline Sample code & $\begin{array}{l}\text { ENR gum for } \\
\text { soft matrix (g) }\end{array}$ & $\begin{array}{l}\text { ENR gum for } \\
\text { hard vitrimer } \\
\text { powders }(\mathrm{g})\end{array}$ & $\begin{array}{l}\text { Total SA in the } \\
\text { sample }(\mathrm{g})\end{array}$ & $\begin{array}{l}\text { SAcontentin hard } \\
\text { vitrimer powders } \\
(w t \%)^{*}\end{array}$ & $\begin{array}{l}\text { MWCNTs } \\
\text { (g) }\end{array}$ \\
\hline hetero-3.8 & 80 & 20 & 3.8 & 9.5 & / \\
\hline hetero-4.75 & 70 & 30 & 4.75 & 9.5 & / \\
\hline hetero-5.7 & 60 & 40 & 5.7 & 9.5 & / \\
\hline hetero- 6.65 & 50 & 50 & 6.65 & 9.5 & / \\
\hline hetero-7.6 & 40 & 60 & 7.6 & 9.5 & / \\
\hline hetero- 8.55 & 30 & 70 & 8.55 & 9.5 & / \\
\hline hetero-9.5 & 20 & 80 & 9.5 & 9.5 & / \\
\hline hetero-H-4.94 & 60 & 40 & 4.94 & 7.6 & / \\
\hline hetero-H-5.7 & 60 & 40 & 5.7 & 9.5 & / \\
\hline hetero-H- 6.46 & 60 & 40 & 6.46 & 11.4 & / \\
\hline hetero-H-7.22 & 60 & 40 & 7.22 & 13.3 & / \\
\hline hetero-H-7.98 & 60 & 40 & 7.98 & 15.2 & / \\
\hline hetero-H-8.74 & 60 & 40 & 8.74 & 17.1 & / \\
\hline hetero-H-9.5 & 60 & 40 & 9.5 & 19 & / \\
\hline hetero-S-7.98 & 20 & 80 & 7.98 & 9.5 & / \\
\hline hetero-S-8.23 & 20 & 80 & 8.23 & 9.5 & / \\
\hline hetero-S-8.87 & 20 & 80 & 8.87 & 9.5 & / \\
\hline hetero-S-9.5 & 20 & 80 & 9.5 & 9.5 & / \\
\hline hetero-9.5-2CNT & 20 & 80 & 9.5 & 9.5 & 2 \\
\hline hetero-9.5-4CNT & 20 & 80 & 9.5 & 9.5 & 4 \\
\hline
\end{tabular}

${ }^{*}$ Relative to the weight of ENR gum in the hard vitrimer powder

Table S2. Cross-linking density, sol fraction and swelling ratio of homo-VEs and hetero-VEs.

\begin{tabular}{|l|l|l|l|}
\hline Sample code & $\begin{array}{l}\text { Cross-linking density } \\
\left(10^{-4} \mathrm{~mol} / \mathrm{cm}^{3}\right)\end{array}$ & $\begin{array}{l}\text { Sol fraction } \\
(\%)\end{array}$ & $\begin{array}{l}\text { Swelling ratio } \\
(\%)\end{array}$ \\
\hline hetero-3.8 & $3.92 \pm 0.08$ & $5.64 \pm 0.79$ & $326 \pm 3$ \\
hetero-4.75 & $4.42 \pm 0.10$ & $4.54 \pm 0.33$ & $308 \pm 3$ \\
hetero-5.7 & $5.91 \pm 0.09$ & $3.97 \pm 0.20$ & $274 \pm 2$ \\
hetero-6.65 & $6.96 \pm 0.16$ & $4.18 \pm 0.19$ & $256 \pm 2$ \\
hetero-7.6 & $7.60 \pm 0.20$ & $3.70 \pm 0.26$ & $247 \pm 2$ \\
hetero-8.55 & $9.88 \pm 0.17$ & $3.48 \pm 0.74$ & $224 \pm 1$ \\
hetero-9.5 & $11.41 \pm 0.09$ & $3.16 \pm 0.29$ & $212 \pm 1$ \\
homo-3.8 & $4.73 \pm 0.09$ & $3.51 \pm 0.11$ & $302 \pm 2$ \\
homo-4.75 & $5.67 \pm 0.10$ & $3.54 \pm 0.43$ & $280 \pm 1$ \\
homo-5.7 & $6.32 \pm 0.10$ & $3.61 \pm 0.24$ & $267 \pm 2$ \\
homo-6.65 & $7.72 \pm 0.06$ & $5.03 \pm 3.12$ & $247 \pm 1$ \\
homo-9.5 & $10.40 \pm 0.36$ & $2.76 \pm 0.26$ & $219 \pm 3$ \\
\hline
\end{tabular}

Table S3. The mechanical properties of all the samples. 


\begin{tabular}{|l|l|l|l|}
\hline Samples & Stress (MPa) & Strain (\%) & Stress at 100\% strain (MPa) \\
\hline homo-3.8 & $3.73 \pm 0.96$ & $225 \pm 39$ & $1.50 \pm 0.06$ \\
homo-4.75 & $4.02 \pm 0.15$ & $208 \pm 5$ & $1.71 \pm 0.02$ \\
homo-5.7 & $4.75 \pm 0.55$ & $200 \pm 16$ & $1.97 \pm 0.03$ \\
homo-6.65 & $6.24 \pm 0.10$ & $199 \pm 3$ & $2.42 \pm 0.03$ \\
homo-9.5 & $7.28 \pm 0.44$ & $166 \pm 3$ & $3.51 \pm 0.05$ \\
hetero-3.8 & $6.78 \pm 0.38$ & $302 \pm 3$ & $1.33 \pm 0.05$ \\
hetero-4.75 & $7.78 \pm 0.95$ & $272 \pm 14$ & $1.65 \pm 0.03$ \\
hetero-5.7 & $11.81 \pm 0.89$ & $264 \pm 1$ & $2.22 \pm 0.05$ \\
hetero-6.65 & $15.53 \pm 0.51$ & $240 \pm 5$ & $3.73 \pm 0.13$ \\
hetero-7.6 & $16.51 \pm 2.64$ & $229 \pm 10$ & $3.94 \pm 0.13$ \\
hetero-8.55 & $18.96 \pm 1.59$ & $219 \pm 5$ & $4.74 \pm 0.16$ \\
hetero-9.5 & $20.60 \pm 1.82$ & $196 \pm 8$ & $6.98 \pm 0.29$ \\
hetero-H-4.94 & $11.49 \pm 1.76$ & $300 \pm 13$ & $1.67 \pm 0.05$ \\
hetero-H-5.7 & $10.60 \pm 0.44$ & $259 \pm 3$ & $2.07 \pm 0.05$ \\
hetero-H-6.46 & $14.31 \pm 0.58$ & $260 \pm 4$ & $2.78 \pm 0.11$ \\
hetero-H-7.22 & $11.73 \pm 1.22$ & $208 \pm 6.4$ & $4.17 \pm 0.39$ \\
hetero-H-7.98 & $13.11 \pm 1.17$ & $186 \pm 5$ & $6.11 \pm 0.25$ \\
hetero-H-8.74 & $14.21 \pm 1.04$ & $168 \pm 5$ & $8.25 \pm 0.47$ \\
hetero-H-9.5 & $13.76 \pm 0.56$ & $151 \pm 8$ & $9.24 \pm 0.47$ \\
hetero-S-7.98 & $12.60 \pm 1.02$ & $186 \pm 7$ & $4.65 \pm 0.39$ \\
hetero-S-8.23 & $15.53 \pm 2.07$ & $202 \pm 10$ & $4.86 \pm 0.20$ \\
hetero-S-8.87 & $16.73 \pm 1.08$ & $193 \pm 3$ & $5.87 \pm 0.13$ \\
hetero-S-9.5 & $16.65 \pm 2.63$ & $184 \pm 9$ & $6.50 \pm 0.39$ \\
hetero-9.5-2CNT & $19.83 \pm 1.11$ & $188 \pm 6$ & $7.38 \pm 0.05$ \\
hetero-9.5-4CNT & $22.30 \pm 2.49$ & $175 \pm 11$ & $11.28 \pm 0.24$ \\
\hline
\end{tabular}

Table S4. Comparison of cross-linking density of hetero-5.7 and hetero-9.5 without and with prestretching.

\begin{tabular}{|l|l|}
\hline Sample code & $\begin{array}{l}\text { Cross-linking density } \\
\left(10^{-4} \mathrm{~mol} / \mathrm{cm}^{3}\right)\end{array}$ \\
\hline $\begin{array}{l}\text { hetero-5.7 without prestretching } \\
\text { hetero-5.7 with prestretching }\end{array}$ & $5.69 \pm 0.11$ \\
hetero-9.5 without prestretching & $5.81 \pm 0.14$ \\
hetero-9.5 with prestretching & $10.82 \pm 0.33$ \\
\hline
\end{tabular}

\section{References}

(1) S. Wu, T. Lin, B. Guo, Elastic-resilience-induced dispersion of carbon nanotubes: a novel way of fabricating high performance elastomer, Nanotechnology 2013, 24, 465708.

(2) Tang, Z.; Liu, Y.; Guo, B.; Zhang, L. Malleable, Mechanically Strong, and Adaptive Elastomers Enabled by Interfacial Exchangeable Bonds. Macromolecules 2017, 50 (19), 7584-7592.

(3) He, C. F.; Christensen, P. R.; Seguin, T. J.; Dailing, E. A.; Wood B. M.; Walde, R. K.; Persson, K. A.; Russell, T, P.; Helms, B. A. Conformational Entropy as a Means to Control the Behavior of Poly(diketoenamine) Vitrimers In and Out of Equilibrium. Angew. Chem. Int. Ed. 2019, 58, 1-6. 
(4) Hayashi, M.; Yano, R; Takasu, A. Synthesis of amorphous low Tg polyesters with multiple COOH side groups and their utilization for elastomeric vitrimers based on post-polymerization cross-linking. Polym. Chem. 2019, 10, 20472056.

(5) Yu, K.; Taynton, P.; Zhang, W.; Dunn, M. L.; Qi, H. J. Influence of stoichiometry on the glass transition and bond exchange reactions in epoxy thermoset polymers. RSC Adv. 2014, 4, 48682-48690.

(6) Qiu, M.; Wu, S.; Fang, S. F.; Tang, Z. H.; Guo, B. C. Sustainable, recyclable and robust elastomers enabled by exchangeable interfacial cross-linking. J. Mater. Chem. A, 2018, 6, 13607-13612.

(7) Huang, J.; Zhang, L.; Tang, Z.; Wu, S.; Guo, B. Reprocessable and robust crosslinked elastomers via interfacial CN transalkylation of pyridinium. Compo. Sci. Technol. 2018, 168, 320-326. 